\title{
Músculos accesorios de la muñeca: Revisión pictográfica en US y RM
}

\author{
Dres. Michael Hirsch $\mathbf{S}^{(1)}$., Pablo Avaria $P^{(2)}$.
}

1. Médico Radiólogo Departamento de Imágenes Clínica Alemana de Temuco. Profesor Asistente Adjunto Facultad de Medicina Clínica Alemana - Universidad del Desarrollo. Temuco - Chile.

2. Residente de Radiología Facultad de Medicina Clínica Alemana - Universidad del Desarrollo. Temuco - Chile.

\section{Accesory muscle of the wrist: A pictographic review}

\begin{abstract}
Although the accessories muscles are part of our nature, to recognize them allows us to avoid confusion with other pathologies. Even some accessories wrist muscles may be symptomatic and require surgical intervention. In this paper we present the anatomy and imaging aspects of ultrasound and magnetic resonance imaging of these muscles, so that the radiologist can become familiar with them, avoiding unnecessary interventions or diagnostic errors. Key words: Accesory muscle, Anatomical variant, Magnetic resonance imaging, Radiology, Ultrasound, Wrist.
\end{abstract}

Resumen: Si bien los músculos accesorios son parte de nuestra naturaleza, el saber reconocerlos nos permite evitar confusiones con otras entidades patológicas. Incluso algunos músculos accesorios en la muñeca pueden ser sintomáticos y requerir intervención quirúrgica. En este trabajo exponemos la anatomía y aspectos imaginológicos en ultrasonido y resonancia magnética de dichos músculos, para que el radiólogo esté familiarizado con ellos, evitando errores diagnóstico o intervenciones innecesarias.

Palabras clave: Imagen por resonancia magnética, Músculos accesorios, Muñeca, Radiología, Ultrasonido, Variante anatómica.

Hirsch M., Avaria P. Músculos accesorios de la muñeca: Revisión pictográfica en US y RM. Rev Chil Radiol 2015; 21(4): 138-143.

Correspondencia: Dr. Michael Hirsch S. / mphirsch@gmail.com

Trabajo recibido el 01 de noviembre de 2015. Aceptado para publicación el 13 de diciembre de 2015.

\section{Introducción}

Las variantes anatómicas son parte de la naturaleza de nuestra anatomía. Pueden consistir en la ausencia de estructuras, estructuras supernumerarias y desviaciones de la localización habitual. Los músculos no son la excepción. Gracias a las distintas técnicas de imagen y la cada vez mejor resolución que obtenemos de ellas, es posible detectar músculos accesorios que antes solo eran reportados en casos quirúrgicos o cadavéricos. Muchos de estos pueden pasar desapercibidos en estudios de imágenes, debido a la similar ecoestructura y patrón fibrilar, densidad e intensidad de señal que los músculos nativos en ultrasonido (US), tomografía computada (TC) y resonancia magnética $(\mathrm{RM})$ respectivamente, por lo que el correcto conocimiento de la anatomía normal y las variantes son factores fundamentales que permiten el adecuado diagnóstico de los músculos accesorios, los cuales suelen ser asintomáticos, pero que en ocasiones pueden verse implicados en patologías, como veremos más adelante ${ }^{(1)}$. Mediante el presente trabajo pretendemos ilustrar sobre la anatomía, aspecto imaginológico y algunos diagnósticos diferenciales de los principales músculos accesorios de la muñeca, con énfasis en el diagnóstico por US y RM, siendo las técnicas que permiten evaluar de mejor manera estas variantes.

\section{Abductor accesorio del dedo meñique (Abductor digiti minimi accessorius) \\ El músculo abductor accesorio del dedo meñi-} que (ADMA) se origina en el aspecto ventral de la fascia antebraquial, cursa anterior a las estructuras neurovasculares del canal ulnar (de Guyon), entre el ligamento transverso del carpo y el ligamento palmar del carpo, uniéndose hacia distal con el músculo abductor del dedo meñique o insertándose directamente en la región ulnar de la base de la falange proximal del meñique ${ }^{(1)}$. La identificación de una imagen de aspecto muscular, superficial a las estructuras neurovasculares ulnares, debe hacer sospechar un ADMA ya que el músculo nativo se origina del pisiforme y no presenta extensión proximal (Figura 1). Inéditamente hemos visto un caso en que el ADMA cursa entre la arteria y nervio ulnar (Figura 1f). Es importante describir la presencia de este músculo ya 
que ha sido asociado a la generación de síndrome del canal ulnar ${ }^{(2-4)}$. Puede causar neuropatía ulnar comprimiendo el nervio contra el ligamento transverso del carpo entre el hueso grande y pisiforme durante su contracción. Una diferencia en el grosor muscular entre pacientes asintomáticos $(1,7 \mathrm{~mm})$ y sintomáticos (4 $\mathrm{mm})$ ha sido reportada, indicado que el grosor muscular tendría relación en la generación de sintomatología ${ }^{(4)}$. Su prevalencia ha sido reportada en hasta $24 \%$ de la población ${ }^{(1)}$, siendo el músculo accesorio de la muñeca más frecuente de encontrar.
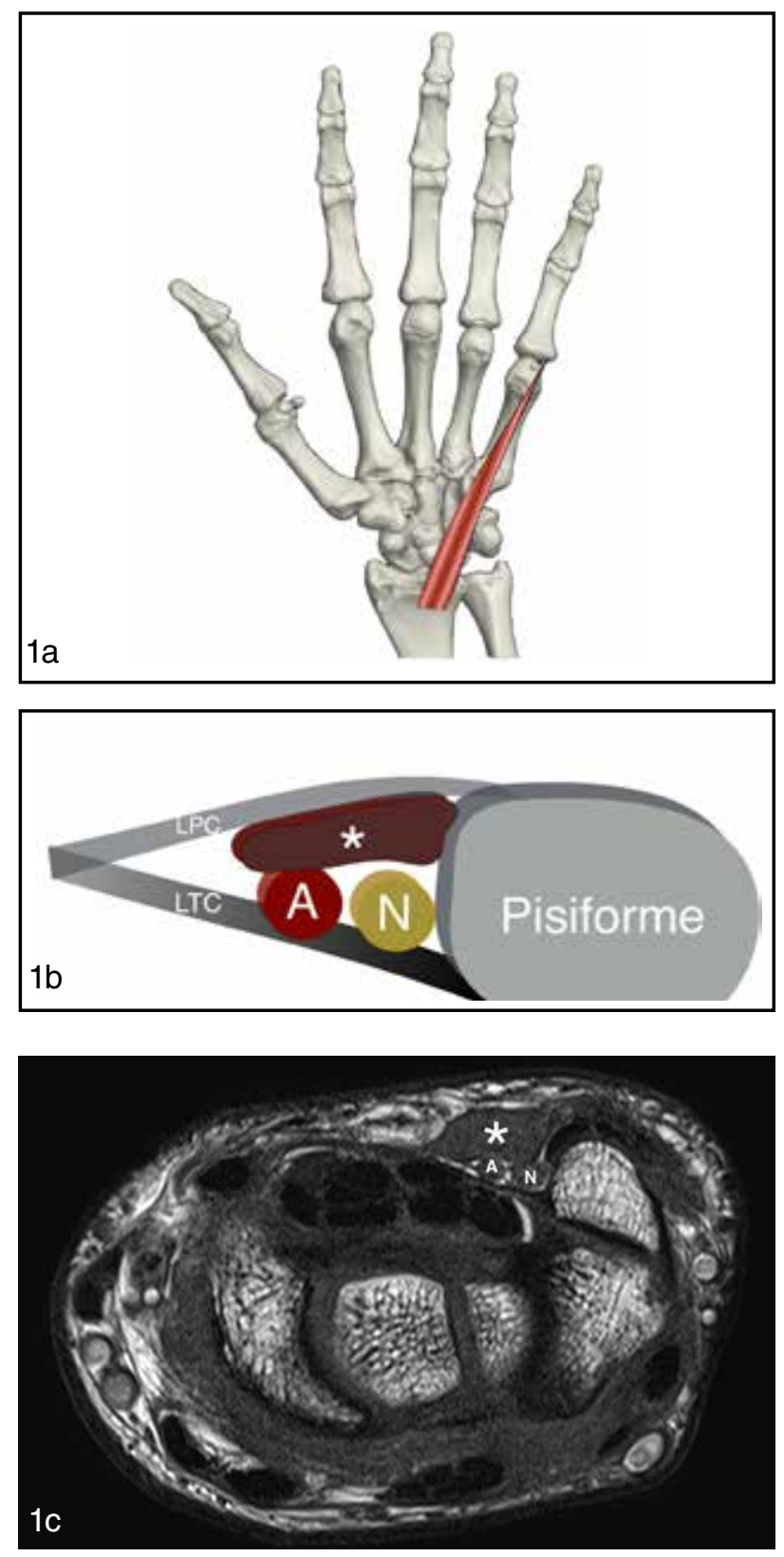

No debe confundirse con el músculo palmar corto, que puede diferenciarse por su ubicación distal al pisiforme, su morfología cuadrangular aplanada y su localización subcutánea en el aspecto proximal de la eminencia hipotenar ${ }^{(1)}$.
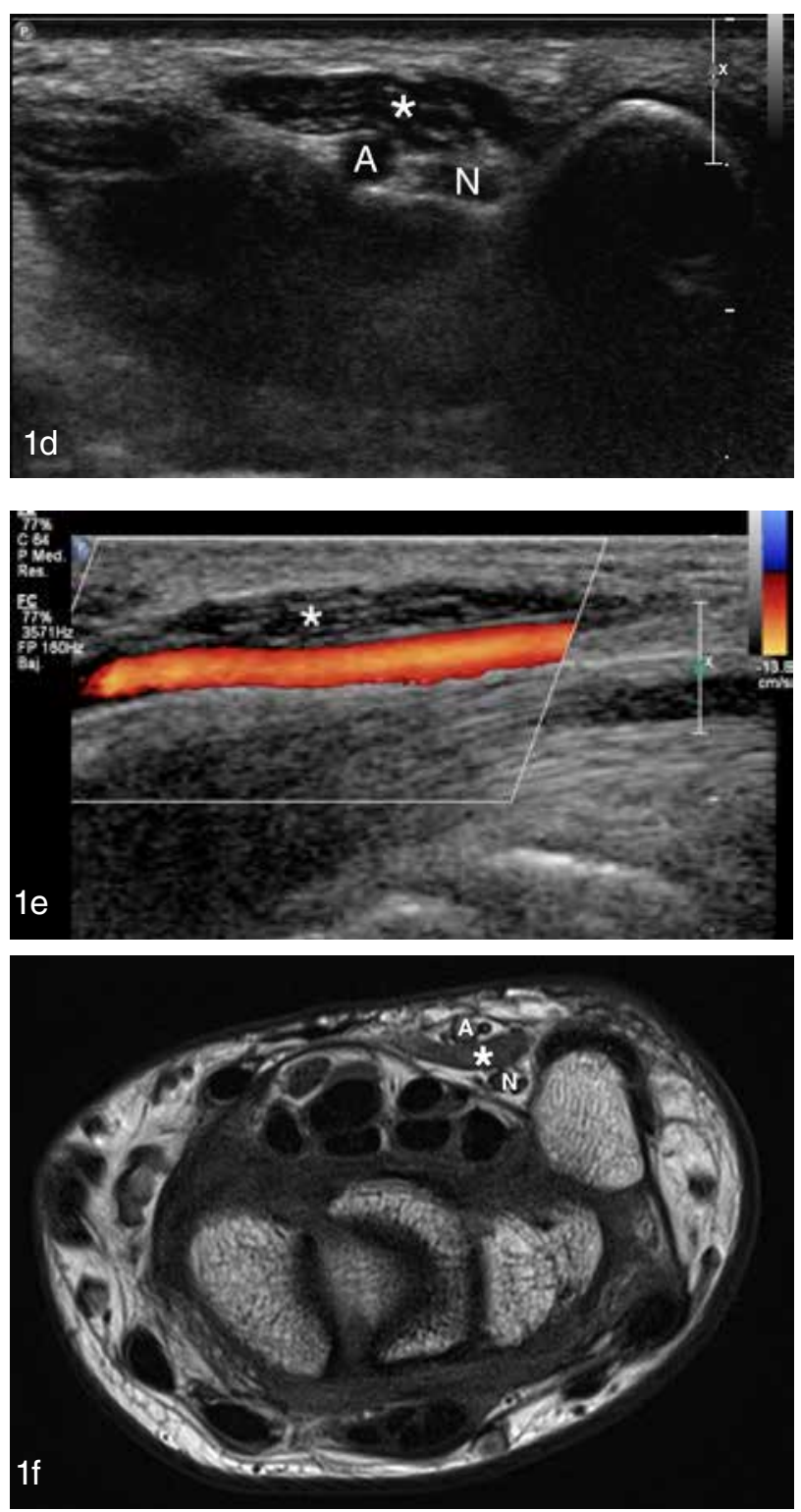

Figura 1. Abductor accesorio del dedo meñique. a) y b) Ilustraciones que ejemplifican el trayecto del músculo, el que se introduce por el canal ulnar (de Guyon) junto a la arteria y nervio ulnar. c) RM y d) US en el plano transversal al nivel del pisiforme y e) US en el plano longitudinal, paralelo a la arteria ulnar. f) RM que muestra extraña variante del músculo, que se lo caliza entre los vasos y nervio ulnar. (asterisco $=$ músculo accesorio, $N=$ nervio ulnar, $A=$ arteria ulnar, $L P C=$ ligamento palmar del carpo, $L T C=$ ligamento transverso del carpo).

\section{Flexor radial corto del carpo (Flexor carpi radialis brevis vel profundus)}

El músculo flexor radial corto del carpo (FCRB) se origina en el aspecto volar del tercio distal del radio, distal al origen del músculo flexor largo del pulgar. Cursa anterior al pronador cuadrado, profundo al flexor radial del carpo y lateral al flexor largo del pulgar, para insertarse en la base del segundo metacarpiano. Su 
inserción está sujeta a variaciones, pudiendo insertarse entre el segundo y cuarto metacarpiano o en la superficie radial de algunos huesos del carpo como el escafoides, trapecio, trapezoide y hueso grande (Figura $2)^{(5,6)}$. Cuando entra a la mano lo hace a través del túnel osteofibroso del flexor radial del carpo. Se han descrito variantes extremadamente infrecuentes, donde se origina desde la metáfisis radial distal, ocupado la posición del pronador cuadrado, causando hipoplasia de éste ${ }^{(5)}$. Puede causar neuropatía compresiva del nervio interóseo anterior, distal a las ramas que inervan el músculo palmar largo y el flexor profundo de los dedos, por lo que rara vez presenta síntomas clínicamente significativos ${ }^{(5)}$. No debe ser confundido con gangliones que habitualmente pueden localizarse en el aspecto volar y lateral del radio distal. El patrón fibrilar, densidad y señal permiten diferenciarlo en US, TC y RM respectivamente de estas lesiones. Se ha reportado tenosinovitis del FCRB, causando dolor, sin embargo, son casos aislados, casi anecdóticos ${ }^{(5)}$. Es un músculo accesorio infrecuente, su incidencia ha sido reportada entre 2,6 y $7,5 \%{ }^{(5)}$.
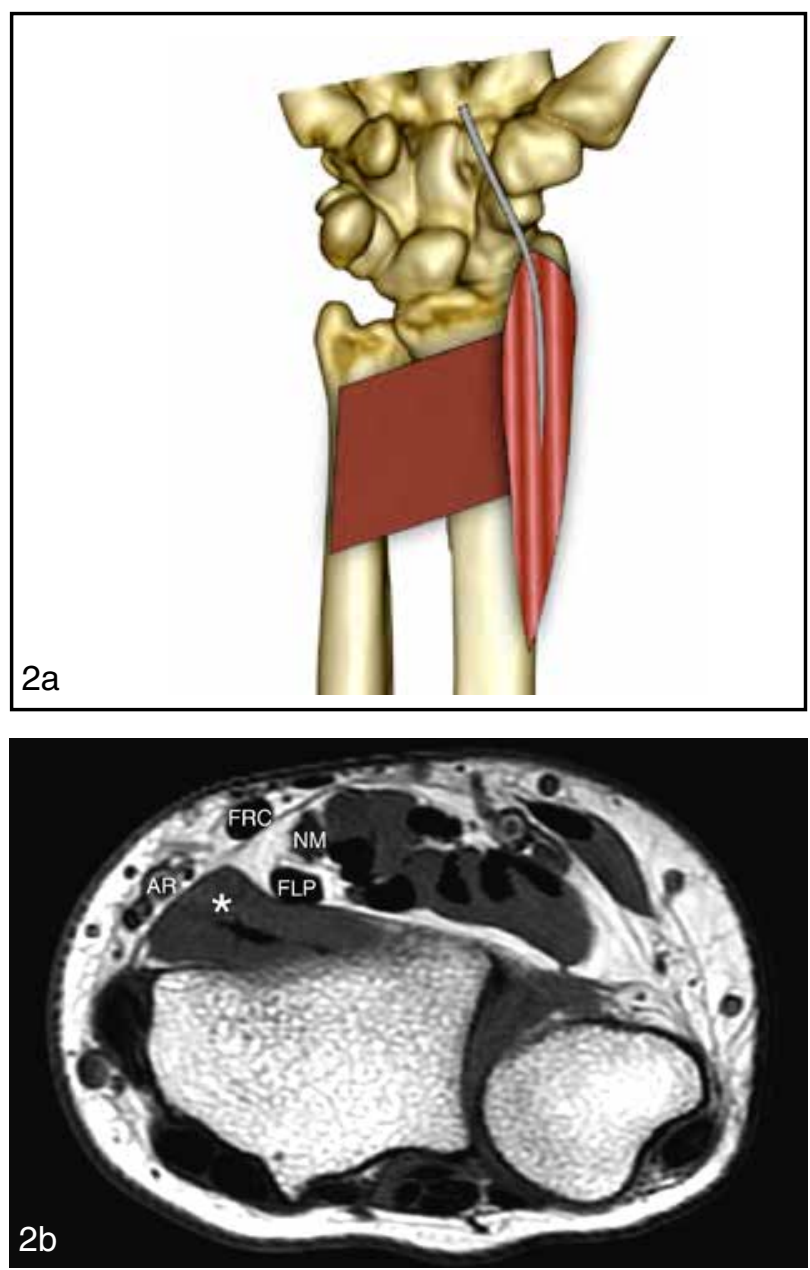
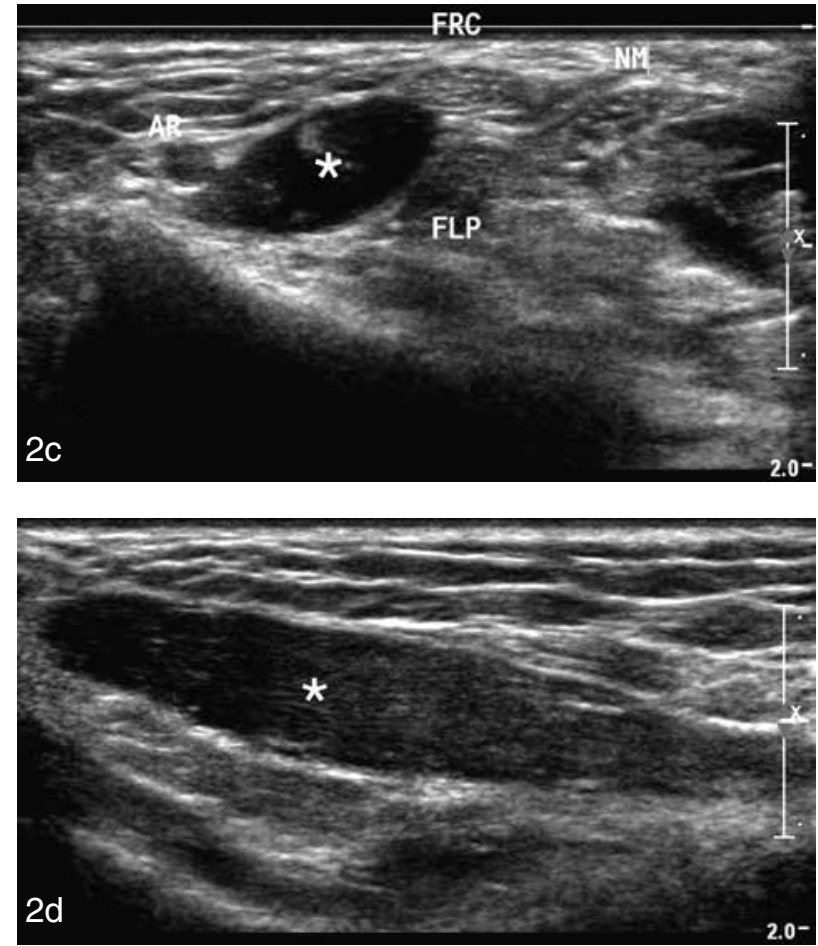

Flexor superficial accesorio del índice (Flexor digitorum superficialis indicis)

El músculo flexor superficial del índice (FDS), se origina del tendón común de los flexores, se extiende por el antebrazo dividiéndose en planos superficiales y profundos antes de entrar al túnel del carpo ${ }^{(1)}$.

EI FDS accesorio, es una variante rara, que se origina del tendón del FDS, adyacente al ligamento transverso del carpo (retináculo flexor) y se inserta típicamente en la región de la polea A1 (cabeza del metatarsiano) ${ }^{(7)}$. Han sido descritas tres principales variantes de este músculo accesorio, incluyendo un vientre muscular localizado completamente en la palma, reemplazando al tendón normal, que puede manifestarse como aumento de volumen ${ }^{(1)}$. Una segunda variante con un músculo digástrico que presenta un vientre en el antebrazo y el otro en la palma pudiendo identificar tejido muscular hacia distal, hasta el segundo metacarpiano ${ }^{(3)}$. Y una tercera forma donde existe un vientre en el antebrazo que se extiende hasta el túnel carpiano, pero no más allá en la palma (Figura 3). Aquellas variantes con efecto de masa por presencia de vientres musculares en la palma, pueden presentarse como síndrome del túnel carpiano $^{(1,4,8,9)}$, particularmente durante la extensión del índice, para lo cual la evaluación dinámica con US se demuestra especialmente útil(4). El examen se debe iniciar con los dedos extendidos, para luego realizar maniobras dinámicas de flexión y extensión

Figura 2. Flexor radial corto del carpo. a) Ilustración que ejemplifica el trayecto del músculo superficial al pronador cuadrado. b) RM y c) US en el plano transversal a nivel del extremo distal del carpo. Obsérvese la localización del músculo entre la arteria radial y el flexor largo del pulgar. d) US en el plano longitudinal. (asterisco= músculo accesorio, AR=arteria radial, $F L P=$ flexor largo del pulgar, $F R C=$ flexor radial del carpo, $N M=$ nervio mediano). 
del índice, evaluando su relación con el túnel carpia$\mathrm{no}^{(4)}$. En el caso de los vientres musculares palmares, estos se localizan entre el tejido adiposo subcutáneo y el segundo metacarpiano. En cortes axiales más proximales se puede observar como el tendón del FDS accesorio se continua con un vientre muscular a nivel de la palma ${ }^{(3)}$. De nuestro conocimiento, no existen estudios de prevalencia que consideren a este músculo.
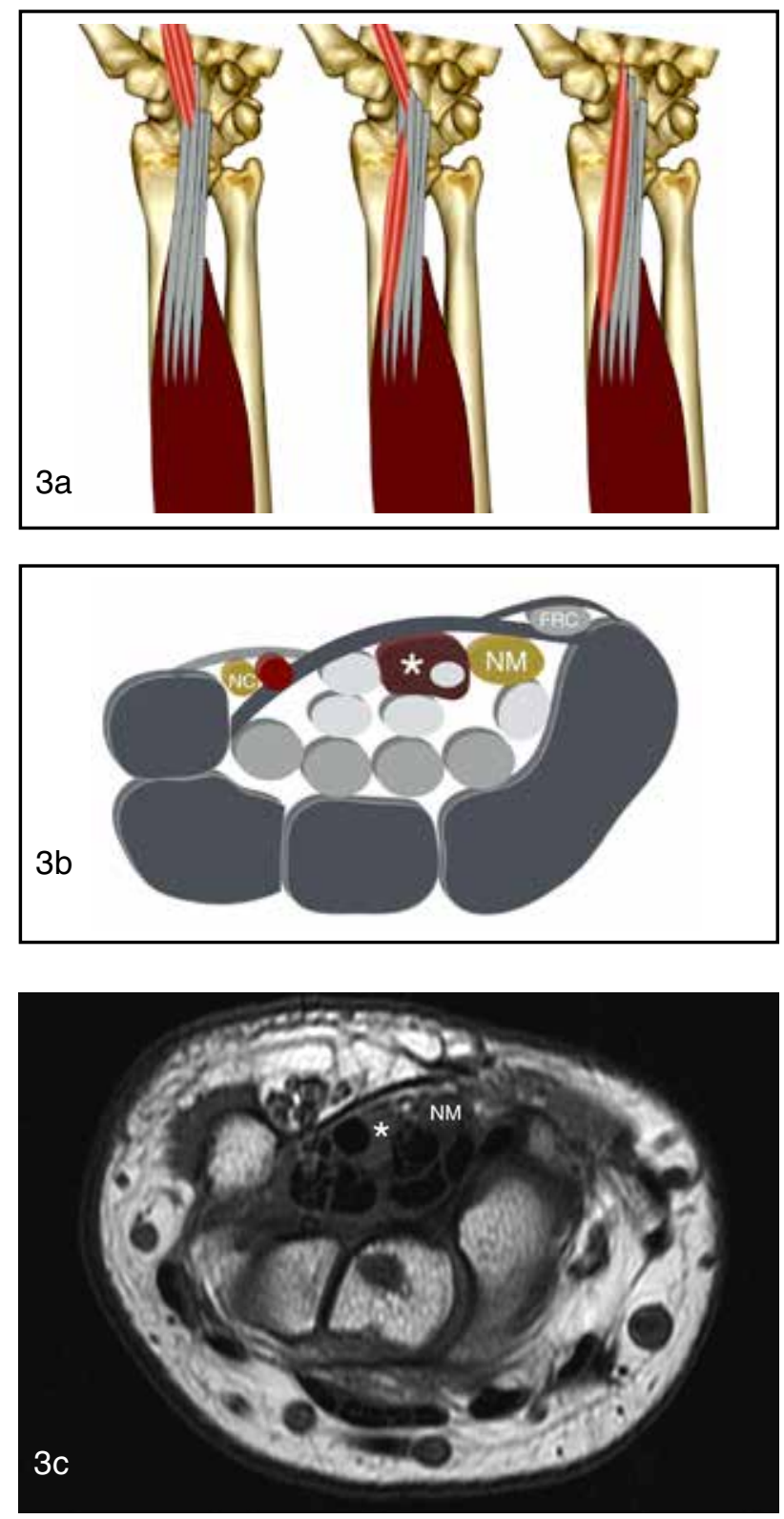

Figura 3. Flexor superficial accesorio del dedo índice. a) Ilustración que ejemplifica las tres variantes descritas sobrepuestas al flexor superficial de los dedos, b) llustración que ejemplifica la tercera variante mostrada en figura $A$, donde se observa el trayecto del músculo, que se introduce al tunel del carpo. c, d) RM y e, f) US en los planos transversal al nivel del pisiforme y longitudinal de esta misma variante. Obsérvese como se introduce el músculo al tunel carpiano y la extensión bajo el ligamento transverso del carpo (flechas). (asterisco= músculo accesorio).
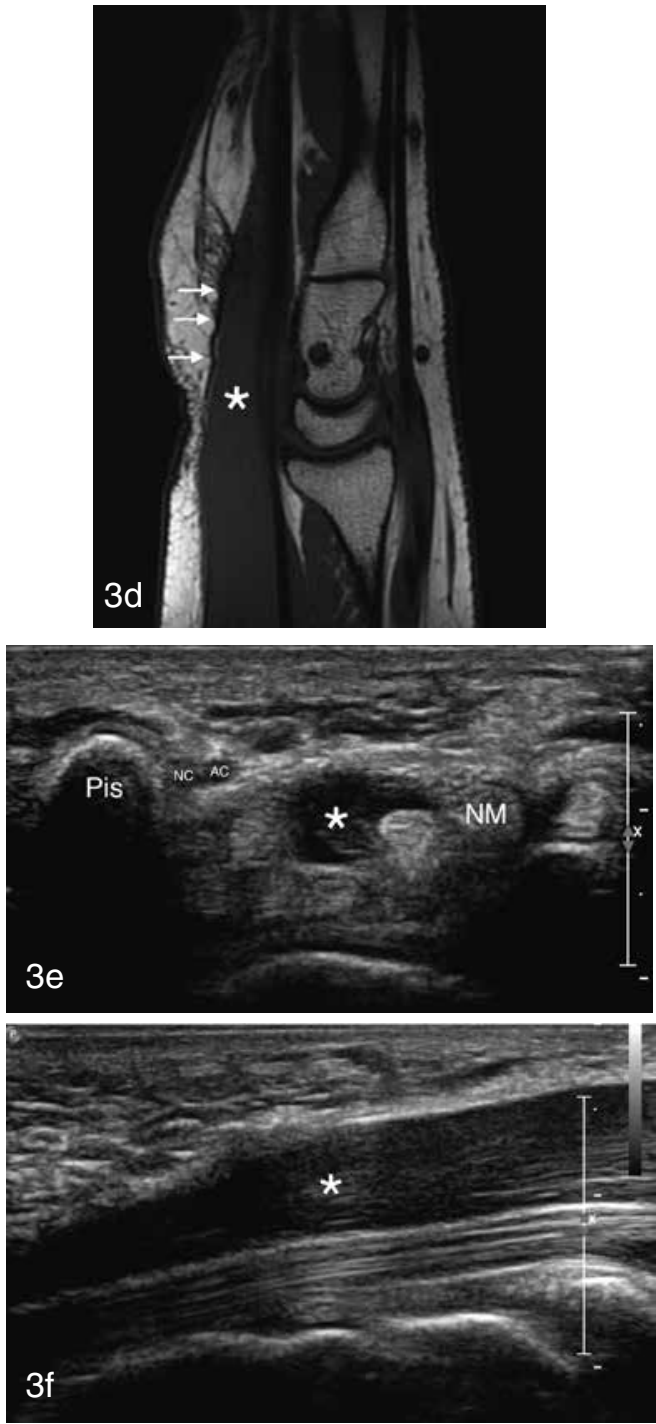

\section{Extensor corto de los dedos de la mano (Extensor digitorum brevis manus)}

El músculo extensor corto de los dedos de la mano (EDBM) presenta un origen variable, siendo el más frecuente, el aspecto dorsal de la capsula articular de la muñeca, profundo al retináculo extensor ${ }^{(4,7)}$. También puede originarse del radio distal o de la fascia carpal profunda. Se inserta típicamente en la expansión del extensor (dosel) del dedo medio o índice, pudiendo insertarse también en el cuarto y quinto dedo ${ }^{(1)}$.

Se observa como un vientre muscular localizado al lado del tendón extensor del índice, profundo a los tendones extensores de los dedos, entre el segundo y tercer metacarpiano, con el vientre muscular habitualmente a nivel de la articulación carpo-metacarpiana (Figura 4). Dentro de su diagnóstico diferencial en imágenes se deben considerar los gangliones dorsales, tenosinovitis y los tumores de células gigantes de la vaina tendínea ${ }^{(1)}$.

Clínicamente se manifiesta como aumento de volumen dorsal, común y erróneamente diagnosticado como ganglión, sinovitis o carpal boss, usualmente indoloro ${ }^{(8,10)}$.

Su prevalencia se ha reportado en $1-3 \%{ }^{(4)}$. 

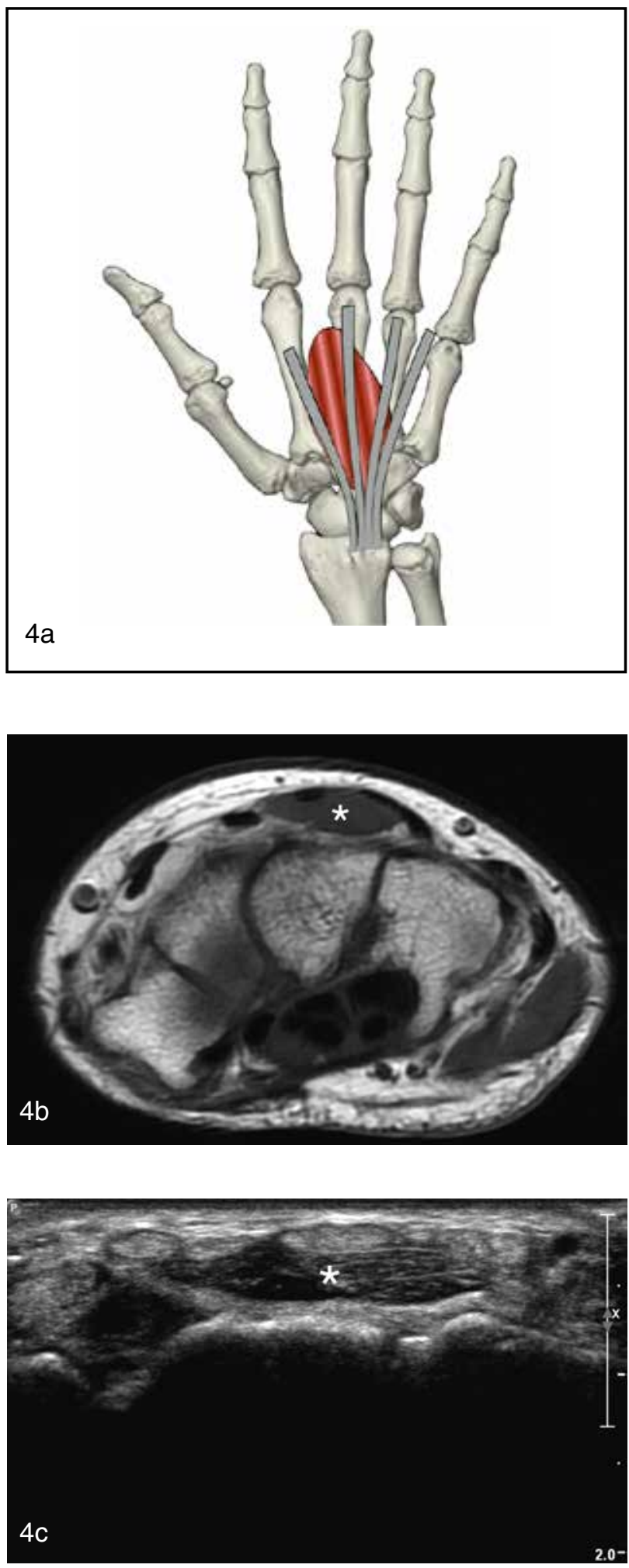

Figura 4. Extensor corto de los dedos de la mano. a) Ilustración que ejemplifica el trayecto del músculo. b) $R M$ y c) US en el plano transversal cercano a la articulación carpo-metacarpiana. Observese el músculo entre los tendones extensores de los dedos y superficie ósea dorsal. d) US en el plano longitudinal demostrando la extensión del músculo sobre los huesos proximales y distales del carpo y base de metacarpianos (Asterisco= músculo accesorio).

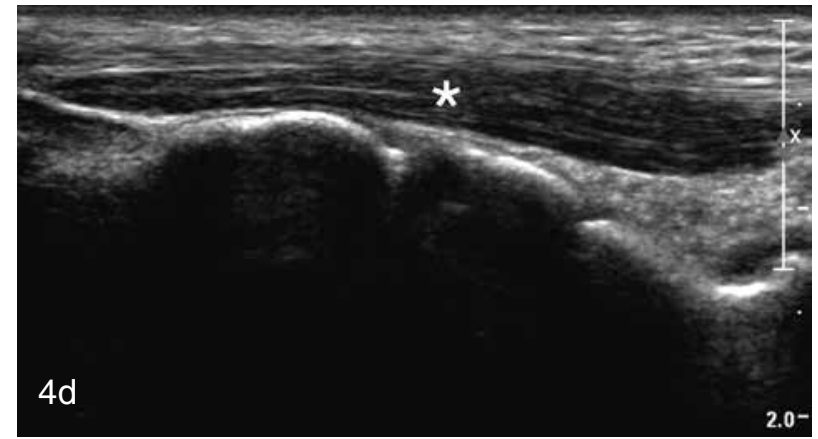

\section{Palmar largo (Palmaris longus)}

El músculo nativo tiene su origen en el tendón conjunto de las flexores y se inserta, en la fascia palmar. Se considera un músculo vestigial de la muñeca y es uno de los con mayor variabilidad en su presentación ${ }^{(3)}$. Puede estar duplicado, ser digástrico, completamente muscular, tendinoso proximal y muscular distal (palmar largo reverso) y como palmar largo accesorio o epifascial, que representa una variante extremadamente rara, se origina en la fascia subcutánea del antebrazo y se inserta en la aponeurosis palmar superficial. El accesorio, digástrico, muscular y reverso, pueden identificarse como tejido muscular en la línea media superficial al retináculo flexor. Este músculo es frecuentemente pasado por alto $^{(1)}$. Clínicamente puede manifestarse como una masa palpable o por compresión del nervio mediano o cubital. Dada su poca relevancia como flexor, muchas veces es utilizado para reparación quirúrgica de roturas tendinosas ${ }^{(2)}$. Su prevalencia se ha reportado en $7 \%$ considerando todas sus presentaciones ${ }^{(1)}$.

\section{Origen proximal de los músculos lumbricales}

Cada uno de los cuatro músculos lumbricales nacen desde sus respectivos tendones del músculo flexor profundo de los dedos, distal al túnel del carpo, insertándose en el aspecto radial de la expansión extensora al nivel la falange proximal correspondiente. Normalmente con la flexión de los dedos, su porción proximal puede migrar dentro del túnel del carpo. En hasta un $22 \%{ }^{(3,4)}$ de los individuos presentan extensión hacia el túnel carpiano, pudiendo manifestarse con un síndrome del túnel del carpo ${ }^{(3,4)}$. Por lo tanto para su correcto diagnostico la muñeca debe examinarse en extensión. Al igual que en los restantes músculos su adecuada evaluación puede realizarse con estudio dinámico con US ${ }^{(4)}$.

\section{Conclusión}

Existe una gran cantidad de músculos accesorios en distintas localizaciones anatómicas y la muñeca no es la excepción. EI US y RM constituyen excelentes métodos para su evaluación, presentando el US la ventaja de la evaluación dinámica. La mayoría de estas estructuras no tienen manifestaciones clínicas 
y son detectadas como hallazgos incidentales. Sin embargo, algunos de ellos pueden ser sintomáticos, por lo que la adecuada correlación clínico-radiológica es fundamental. Dentro de las formas de presentación más frecuentes, se encuentran las masas palpables y las neuropatías compresivas, en especial aquellas en relación al túnel carpiano y canal cubital. Un acabado conocimiento de la anatomía, el conocimiento de estas estructuras y un alto índice de sospecha, pueden permitir al radiólogo plantear la presencia de un músculo accesorio y diferenciarlos de otros diagnósticos diferenciales.

\section{Bibliografía}

1. Sookur PA, Naraghi AM, Bleakney RR, Jalan R, Chan O, White LM. Accessory muscles: Anatomy, symptoms, and radiologic evaluation. Radiographics 2008; 28(2): 481-499.

2. Spiess AM, Gursel E. Entrapment of the ulnar nerve at Guyon's canal by an accessory abductor digiti minimi muscle. Plast Reconstr Surg 2006; 117(3): 1060-1061.

3. Timins ME. Muscular anatomic variants of the wrist and hand: findings on MR imaging. AJR Am J Roentgenol 1999; 172(5): 1397-1401.
4. Bianchi S, Martinoli C. Wrist. In: Baert A, Knauth M, Sartor K, eds. Ultrasound of the Musculoskeletal System. 2007 ed. Germany: Springer Berlin Heidelberg 2007; 425-494.

5. Lee YM, Song SW, Sur YJ, Ahn CY. Flexor carpi radialis brevis: An unusual anomalous muscle of the wrist. Clin Orthop Surg 2014; 6(3): 361-364.

6. Nakahashi T, Izumi R. Anomalous interconnection between flexor and extensor carpi radialis brevis tendons. Anat Rec 1987; 218(1): 94-97.

7. 7. Elias LS, Schulter-Ellis FP. Anomalous flexor superficialis indicis: Two case reports and literature review. J Hand Surg Am 1985; 10(2): 296-299.

8. Anderson MW, Benedetti P, Walter J, Steinberg DR. MR appearance of the extensor digitorum manus brevis muscle: A pseudotumor of the hand. AJR Am J Roentgenol 1995; 164(6): 1477-1479.

9. Stephens N, Marques E, Livingston C. Anomalous flexor digitorum superficialis muscle belly presenting as a mass within the palm. Can J Plast Surg 2007; 15(1): 44-46.

10. Rodríguez-Niedenfuhr M, Vázquez T, Golano P, Parkin I, Sanudo JR. Extensor digitorum brevis manus: Anatomical, radiological and clinical relevance. A review. Clin Anat 2002; 15(4): 286-292.

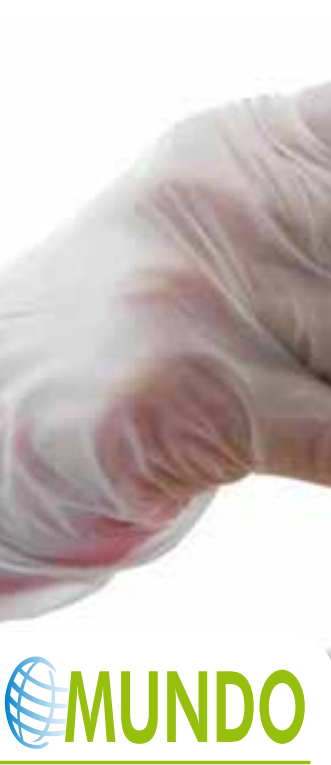

UN MUNDO DE SOLUCIONES EN IMAGEN

GEL ULTRASONIDO BIO - HEALTH

NUEVA FÓRMULA

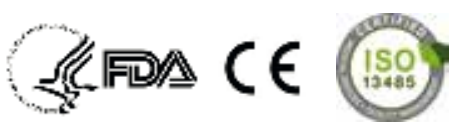

